\title{
Survival and NF1 Analysis in a Cohort of Orthopedics Patients with Malignant Peripheral Nerve Sheath Tumors
}

\author{
Daniel K. Knewitz, ${ }^{1}$ Colin J. Anderson, ${ }^{2,3}$ William T. Presley, ${ }^{4}$ MaryBeth Horodyski, ${ }^{2}$ \\ Mark T. Scarborough, ${ }^{2,5}$ and Margaret R. Wallace $\mathbb{i D}^{4,5,6}$ \\ ${ }^{1}$ University of Florida College of Medicine, MS3, Gainesville, FL, USA \\ ${ }^{2}$ Department of Orthopaedics and Rehabilitation, University of Florida College of Medicine, Gainesville, FL, USA \\ ${ }^{3}$ Department of Orthopedic Surgery, Levine Cancer Institute and Musculoskeletal Institute, \\ Carolinas Medical Center-Atrium Health, Charlotte, NC, USA \\ ${ }^{4}$ Department of Molecular Genetics \& Microbiology, University of Florida College of Medicine, Gainesville, FL, USA \\ ${ }^{5}$ University of Florida Health Cancer Center, Gainesville, FL, USA \\ ${ }^{6}$ University of Florida Genetics Institute, Gainesville, FL, USA
}

Correspondence should be addressed to Margaret R. Wallace; peggyw@ufl.edu

Received 6 April 2021; Accepted 18 September 2021; Published 4 October 2021

Academic Editor: Kanya Honoki

Copyright (c) 2021 Daniel K. Knewitz et al. This is an open access article distributed under the Creative Commons Attribution License, which permits unrestricted use, distribution, and reproduction in any medium, provided the original work is properly cited.

\begin{abstract}
Neurofibromatosis type 1 (NF1) is an autosomal dominant tumor syndrome in which benign plexiform neurofibromas are at risk of transforming into malignant peripheral nerve sheath tumors (MPNSTs), a very rare soft-tissue sarcoma. The prognosis of patients with MPNSTs is poor, with most studies reporting $<50 \%$ survival at five years. However, studies evaluating MPNSTs are limited and report heterogeneous results. Because no MPNST-specific evidence-based treatment guideline exists, individual institutional experiences are very informative to the field. The main objective of this study was to investigate and report MPNST prognostic clinical and genetic biomarkers from our institution's Orthopedics service experience treating 20 cases from 1992 to 2017. Most patients were treated with resection and adjuvant radiation. Extended follow-up, averaging 11.4 years (ranging 1.1 to 25.1), revealed excellent five-year survival rates: $70 \%$ for overall and 60\% for metastatic disease. An S100 B immunonegative tumor phenotype was associated with a significantly worse outcome than MPNSTs with positive S100 B stain. In addition, NF1 gene mutation analysis was performed on 27 families with NF1 in which at least one affected family member developed MPNSTs. Of the 27 NF1 germline mutations, five were large deletions spanning (or nearly spanning) the gene (18.5\%), substantially more than such deletions in NF1 in general, consistent with increased risk of MPNSTs in such cases.
\end{abstract}

\section{Introduction}

Neurofibromatosis type 1 (NF1) is an incurable progressive autosomal dominant disease. Despite an NF1 incidence of 1 in 3000 people, the first effective systemic therapy with the MEK inhibitor selumetinib was only recently FDA approved, in 2020 [1]. NF1 is caused by heterozygous germline mutations in the NF1 gene, of which over 5000 have been identified spanning the large locus. The clinical hallmark of NF1 is the neurofibroma, a benign peripheral nerve sheath Schwann-cell tumor. Patients can develop few to thousands of neurofibromas in their lifetimes. Neurofibromas are classified as "cutaneous" when they are superficial, involving nerve endings in the skin; larger and usually deeper "plexiform neurofibromas" involve peripheral nerves [2]. Plexiform neurofibromas are thought to be congenital in origin and occur in about 50\% of patients [2]. Plexiform neurofibromas can grow quite large and are estimated to have a $10-30 \%$ risk of malignant transformation into a malignant peripheral nerve sheath tumor-a rare type of soft-tissue sarcoma-particularly if they have a nodular phenotype [2]. MPNSTs are rare, affecting only 1.4 in 
100,000 people, with half of such cases occurring in NF1 patients [3]. MPNSTs are not reported to show a predilection toward a specific anatomical region of the body. Cutaneous cases of MPNSTs have been described, but they do not typically occur in NF1 patients [4]. Overall, the lifetime risk of MPNSTs in NF1 patients is estimated at $12-15 \%$ [5]. In reported studies, the MPNST 5-year survival rate is usually reported as less than 50\% [6] and thus the prognosis is generally considered poor.

Because of its rarity, there have been few clinical trials specifically for patients with MPNSTs and information about natural history and response to therapies in the literature is sparse. Typically, MPNSTs are managed with treatment protocols for soft-tissue sarcomas, as protocols specific to MPNSTs have not been established [7]. Early surgery has been shown to be an effective treatment [8] with a goal of resecting the tumor with wide or negative margins. Adjuvant radiation or chemotherapy regimens have also been used at different institutions to reduce the risk of local recurrence or to treat systemic disease [7]. Although recent trials with novel targeted therapies have so far proven ineffective, recurrent gene mutations that have been associated with more aggressive tumors in patients with NF1, such as the polycomb repressive complex 2 (PRC2) core components, embryonic ectoderm development protein (EED), and suppressor of zeste 12 homolog (SUZ12), may serve as future treatment targets [9-12]. There is some controversy in the literature regarding germline NF1 mutation effect on the risk of developing an MPNST. Some studies reported a higher incidence of deletions spanning the entire NF1 locus and surrounding genes in NF1 patients who develop an MPNST than the NF1 population overall [13], while other reports did not find such a trend [14].

There are a limited number of publications reporting MPNST survival, most with small case numbers and variable outcomes, not unexpected for such a rare tumor. Thus, experience from institutions managing patients with MPNSTs is important to add to the field's knowledge [15]. The purpose of our study was to evaluate both clinical and NF1 mutation data from the MPNST population at our institution to identify prognostic factors.

\section{Materials and Methods}

2.1. Clinical and Demographic Data Collection. As approved by the University of Florida Institutional Review Board, a retrospective medical chart review of 1992 to 2017 was performed from 1992 to 2017 to gather data on our institution's Division of Orthopedic Oncology's experience of treating patients with MPNSTs. The data were extracted from an EPIC electronic medical record service and from the Enneking/Anspach Research Center database in the Department of Orthopedics and Rehabilitation. Inclusion criteria were (1) diagnosis of MPNSTs, (2) definitive treatment performed at our institution, and (3) at least 12 months of follow-up data. Demographic and clinical information collection included gender, self-reported ethnic group, age at initial diagnosis, tumor stage at first diagnosis, time to recurrence, diagnosis of NF1, family history of NF1, therapeutic history, immunohistochemical S100 B data from a pathology service, and survival status.

2.2. NF1 Gene Mutation Analysis. NF1 mutation analysis was performed on DNA samples from the IRB-approved Wallace Genetics Bank from 27 NF1 families bearing at least one MPNST occurrence. Previously existing data included some NF1 cDNA Sanger sequencing, while current testing included PCR and sequencing of 13 exons $(4,5,11,13,14,16$, $18,20,22,26,28,32,40$, and 46 ) of the 57 exons in the locus (NCBI gene sequence: NG_009018.1) (methods described in $[16,17])$. In addition, all samples were screened for large deletions using four TaqMan NF1 copy-number assays across the gene (ThermoFisher; Hs05477010, Hs06413401, Hs05512625, and Hs03960106) (Center for Pharmacogenomics Core, UF Clinical and Translational Institute).

2.3. Statistical Analysis. All data were analyzed using statistical software (SPSS 25, IBM Co. Armonk, NY). The significance level for analyses was set a priori at $p<0.05$. Descriptive statistics were computed for demographic and clinical variables. Kaplan-Meier survival curves were generated to assess the overall survival of the study population and compare between demographic, clinical, survival, and pathological variables.

\section{Results}

A total of twenty subjects (14 males and 6 females) qualified for this study's retrospective chart review. Demographic characteristics are provided in Table 1.

With respect to race, 12,6 , and 1 subject(s) were Caucasian, black, and Hispanic, respectively. Race was not identified in the medical record of one subject. The mean total length of follow-up was 136.9 months. The survival rate for the entire study population was approximately $70 \%$ (Figure 1).

Two subjects had local recurrence, and five had subsequent metastasis. The mean time to local recurrence and time to metastasis were 134.9 and 113.6 months, respectively. Both subjects with local recurrence received radiation therapy. At the last follow-up for all subjects, 14 were alive and 6 were deceased. Two of the five subjects who developed subsequent metastasis were alive at the last follow-up (2018) and survived an average of 197.9 months (range 85.7 to 309.8) after diagnosis of metastasis. Four of these metastases targeted the lung, and one subject developed metastasis in the brain. The five-year survival rate of patients with metastatic disease was $60 \%$.

The female gender was associated with better cumulative survival outcomes, and the difference was not significant $(p=0.45)$. Black subjects were found to have better cumulative survival outcomes than Caucasian and Hispanic subjects; however, the difference was not significant $(p=0.25)$. The most common primary MPNST locations in this patient population were the lower extremities $(n=11)$ and the upper extremities $(n=8)$, followed by the neck $(n=1)$. Six subjects had a diagnosis of NF1. Five subjects 
TABLE 1: Subject demographics and clinical survival information.

\begin{tabular}{|c|c|c|c|c|c|}
\hline & Mean & Std. dev. & Range & Minimum & Maximum \\
\hline Age at diagnosis (years) & 43.4 & 18.4 & 75.4 & 6.0 & 81.4 \\
\hline Time to local recurrence or last follow-up (months) & 134.9 & 102.1 & 309.9 & 4.9 & 314.8 \\
\hline Time to metastasis or last follow-up (months) & 113.6 & 100.0 & 284.8 & 4.9 & 289.7 \\
\hline Total follow-up (months) & 136.9 & 99.8 & 301.1 & 13.7 & 314.8 \\
\hline
\end{tabular}

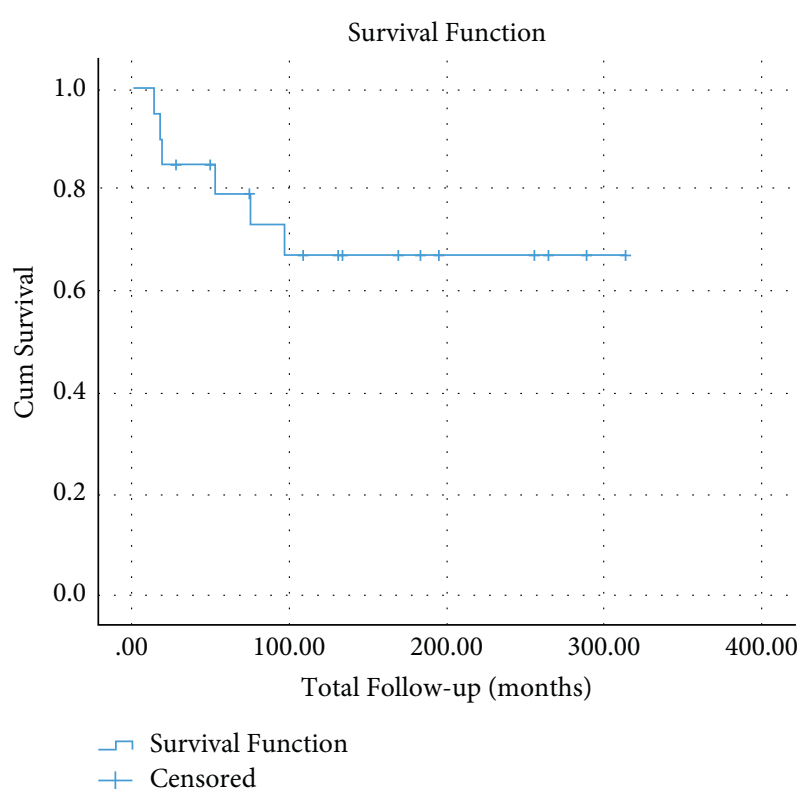

Figure 1: Kaplan-Meier survival curve for all 20 subjects. The 5year survival was approximately $70 \%$.

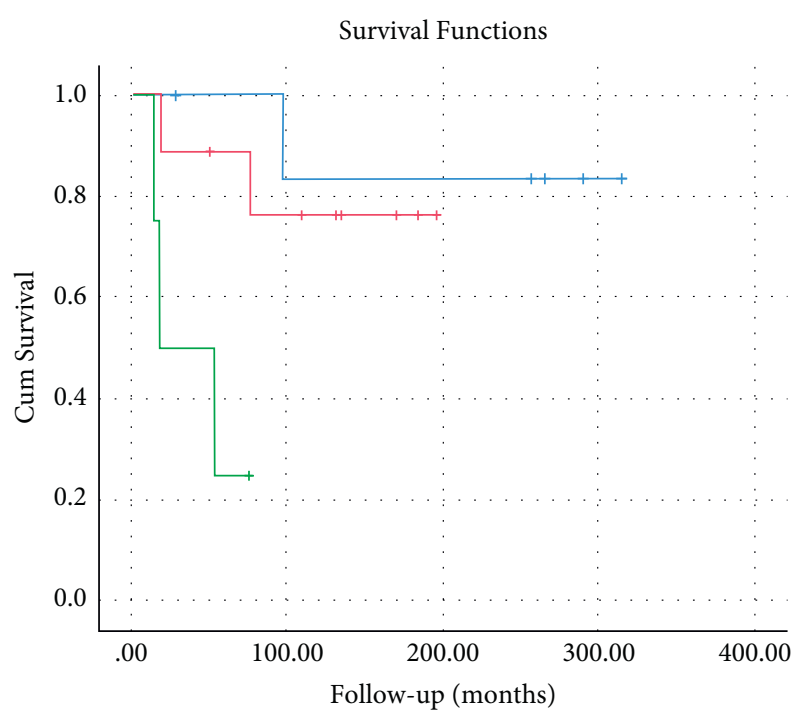

$$
\begin{array}{ll}
\text { S100B Data } & \\
\neg \text { undetermined } & + \text { undetermined-censored } \\
\neg \text { yes } & + \text { yes-censored } \\
\neg \text { no } & + \text { no-censored }
\end{array}
$$

Figure 2: Kaplan-Meier survival curve based on MPNST S100 B status. S100B-negative MPNSTs (green line, $n=4$ ) were associated with a significantly lower survival than S100B-positive tumors (red line, $n=9)(p<0.003)$. were NF1-negative, and the NF1 status for nine subjects was indeterminate. Although subjects with negative NF1 status were found to have better cumulative survival outcomes, the difference was not significant $(p=0.39)$.

Tumor-grade classifications were low $(n=2)$, intermediate $(n=3)$, and high $(n=15)$. Low-grade tumors were found to have better cumulative survival than intermediate and high, although the difference was not significant $(p=0.63)$. AJCC 8th Education Stage classification of the patients was as follows: IA $=2, \mathrm{II}=4, \mathrm{IIIA}=8, \mathrm{IIIB}=6$. Patients with stage IA and II tumors were all alive at the last follow-up. Overall comparison between stages failed to reveal a significant difference in cumulative survival $(p=0.26)$. S100 B immunohistochemistry status was reported positive in 9 tumors, negative in 4 , and could not be determined in 7. Subjects with positive S100 B status were shown to have a significantly better cumulative survival outcome $(p=0.003)$ (Figure 2).

Three subjects were treated with resection alone (1 subsequently developed metastasis), 15 were treated with resection and adjuvant radiation, and two subjects were treated with resection and chemotherapy. Only one of the resections involved an amputation. When comparing cumulative survival in subjects who received chemotherapy $(n=2)$ against subjects who did not receive chemotherapy $(n=18)$, the two subjects receiving chemotherapy had a poorer outcome, although it was nonsignificant $(p=0.69)$. When comparing estimated overall survival in subjects who received radiation (234.1 months) against those who did not (193.9 months), subjects who received radiation had better survival, but the difference was not significant $(p=0.89)$.

Among banked DNAs, there were 27 NF1 cases who developed MPNSTs and/or had a positive family history of MPNSTs. As shown in Table 2, germline NF1 mutations among these 27 families were distributed as follows: nonsense (9), missense (4), frameshift (5), splicing (5), deletion spanning most of the gene (1), and whole-gene deletion (4).

\section{Discussion}

The purpose of this study was to evaluate clinical prognostic factors and genomic biomarkers in patients with MPNSTs treated at our institution. As an academic tertiary-care institution, our institution is a referral center for cancer patients, including those with MPNSTs, from throughout the US as well as internationally. Most similar studies have reported less than a 50\% five-year MPNST survival rate [18-30]. Our cohort had a mean follow-up time of 11.4 years and a $70 \%$ five-year survival rate, the latter of which is superior to any previously reported studies (range 38.3-62.5 years) $[18,19,22-30]$. 
TABLE 2: MPNST-related NF1 germline mutations.

\begin{tabular}{lcc}
\hline Mutation & Effect & Exon \\
\hline c.499delTGTT & Frameshift & 5 \\
c.958 G > Cp.Ala320Pro & Missense & 9 \\
c.1039 C > Tp.Gln347X & Nonsense & 9 \\
c.1782delAG & Frameshift & 16 \\
c.2325 G > A & Splice error & 19 \\
c.2352 G > Ap.Trp784X & Nonsense & 20 \\
c.2446 C > Tp.Arg816X & Nonsense & 21 \\
c.2534insG & Frameshift & 21 \\
c.2540 T > Cp.Leu847Pro & Missense & 21 \\
c.2991-1G > A & Splice error & 23 \\
c.3113+1G > C & Splice error & 23 \\
c.3456_3457insA & Frameshift & 26 \\
C. 3683 delC & Frameshift & 27 \\
c.3826 C > Tp.Arg1276X & Nonsense & 28 \\
c.4255 A > Tp.Lys1419X & Nonsense & 32 \\
c.4435 A > G & Splice error & 34 \\
c.4868 A > Tp.Asn1623Val & Missense & 37 \\
c.5242 C > Tp.Arg1748X & Nonsense & 38 \\
c.5914 C > Tp.Gln1981X & Nonsense & 40 \\
c.6148 C > Tp.Gln2050X & Nonsense & 42 \\
c.6302 C > Gp.Thr2101Arg & Missense & 42 \\
c.7285 C > Tp.Arg2429X & Nonsense & 50 \\
Whole-gene deletion $(n=4)$ & Null & All \\
Large intragenic gene deletion $(n=1)$ & Likely null & $2-50$ \\
\hline$n=27$. NCBI RefSeq: NM 0000267. & &
\end{tabular}

Consistent with previous reports, we noted that subjects with S100B-positive tumors had significantly better outcomes than those with S100B-negative tumors [12]. S100 B is a mature Schwann-cell protein, and loss of immunostaining is consistent with poorer cell differentiation. Further investigation is needed to evaluate the use of this phenotype to stratify patient risk, along with immunostaining of other markers in MPNSTs that are of recent interest such as polycomb repressor complex 2 epigenetic mark H3K27me3 [31] and HMGA2 [32].

The frequency of whole-gene deletions (14.8\%) in the 27 germline NF1 mutations associated with MPNSTs was higher in our population than the $4-5 \%$ rate of such deletions in NF1 patients in general [33]. This frequency is even greater when including samples with a deletion of most of the gene, extending beyond the $3^{\prime}$ end for an unknown distance. Our result is consistent with previous reports suggesting that individuals with such deletions are at a greater risk for MPNSTs [13, 34, 35]. However, this elevated risk could be related to the increased plexiform neurofibroma burden of many such patients [33].

Although a significant difference in survival was not found based on presence of an NF1 diagnosis, patients with NF1 trended toward a poorer outcome, particularly males: one was still alive at the last follow-up at 314.8 months after metastasis, but three (including one with metastatic disease) had survival under five years (52.43 months after metastasis, 13.74 months after diagnosis, and 17.23 months after diagnosis, respectively). The two NF1 female subjects are both still alive (108.78 months after metastasis and 256.61 months after diagnosis). This study was underpowered to detect relationships between race, gender, and other factors with respect to survival. Similar studies with larger cohorts found individual variables associated with survival, such as tumor size $[18,19,22,29]$, margin status [18, 24-28], NF1 diagnostic status $[19,22,26,29,30]$, and tumor grade $[19,21,22,28]$.

Our study had a number of limitations, the foremost being sample size and another being lack of NF1 status in earlier cases. Although a larger sample size might aid in identifying additional significant prognostic factors, it is important to report individual institution experiences because of the lack of evidence-based established therapeutic regimens for MPNST. Information from institutions with better outcomes may be very useful to other groups. Toward this goal, we excluded patients who had received prior MPNST treatment at outside institutions. Although this allowed evaluation of survival outcomes based solely on care at our institution, a more varied subject group might have been more representative of the overall US MPNST patient population experience.

\section{Conclusions}

Given the rarity and lack of clinical trial data for MPNSTs, treatment parameters and survival data from multiple institutions are needed to move toward improved treatment and prognosis. It is critical that patients with rare tumors such as MPNSTs be referred to institutions experienced with such cancers. Our institutional experience with MPNST cases treated in the Orthopedics oncology service, with treatment favoring surgical resection and adjuvant radiation, showed an outstanding five-year survival of $70 \%$. This work also highlighted the potential use of S100 B immunostaining in prognosis. In addition, our study added to the evidence that whole-gene germline NF1 deletions are associated with an increased risk of MPNST.

\section{Data Availability}

This study utilized data derived from patient records at our institution; hence, the data are not available for public release. All pertinent HIPAA-compliant data are published in the accompanying tables and figures.

\section{Conflicts of Interest}

The authors declare that there are no conflicts of interest with regard to this study.

\section{Acknowledgments}

The authors thank the University of Florida College of Medicine's Medical Student Research Program for DKK's support while performing this work. The mutation analysis work was supported through Neurofibromatosis Therapeutic Acceleration Program (NTAP) funding to MRW. The authors also thank Dr. Hua Li for technical assistance; her contribution and that of MRW were in part also supported by a DOD grant to MRW (Award \#W81XWH-20-1-0355). 


\section{References}

[1] First Drug Approved for NF, "Children's tumor foundation," 2018, https://www.ctf.org/first-drug-approved-for-nf.

[2] D. H. Gutmann, R. E. Ferner, and R. H. Listernick, "Neurofibromatosis type 1," Nature Reviews Disease Primers, vol. 3, Article ID 17004, 2017.

[3] J. E. Bates, C. R. Peterson, S. Dhakal, E. J. Giampoli, and L. S. Constine, "Malignant peripheral nerve sheath tumors (MPNST): a SEER analysis of incidence across the age spectrum and therapeutic interventions in the pediatric population," Pediatric Blood and Cancer, vol. 61, no. 11, pp. 1955-1960, 2014.

[4] B. Luzar and G. Falconieri, "Cutaneous malignant peripheral nerve sheath tumor," Surgical Pathology Clinics, vol. 10, no. 2, pp. 337-343, 2017.

[5] E. Uusitalo, J. Leppävirta, A. Koffert et al., "Incidence and mortality of neurofibromatosis: a total population study in Finland," Journal of Investigative Dermatology, vol. 135, no. 3, pp. 904-906, 2015.

[6] J. F. Longo, S. N. Brosius, L. Black et al., "ErbB4 promotes malignant peripheral nerve sheath tumor pathogenesis via Ras-independent mechanisms," Cell Communication and Signaling: CCS, vol. 17, p. 74, Article ID 74, 2019.

[7] D. Bradford and A. Kim, "Current treatment options for malignant peripheral nerve sheath tumors," Current Treatment Options in Oncology, vol. 16, p. 328, Article ID 12, 2015.

[8] Z. C. Liao, C. Zhang, and X. Y. Liu, "Targeted therapy for malignant peripheral nerve sheath tumor: translational research and clinical application," Zhonghua Zhongliu Zazhi, vol. 41, no. 9, pp. 648-653, 2019.

[9] L. M. Kayes, W. Burke, and V. M. Riccardi, "Deletions spanning the neurofibromatosis 1 gene: identification and phenotype of five patients," The American Journal of Human Genetics, vol. 45, no. 3, pp. 424-436, 1994.

[10] M. H. Cnossen, M. N. Van Der Est, M. H. Breuning et al., "Deletions spanning the neurofibromatosis type 1 gene: implications for genotype-phenotype correlations in neurofibromatosis type 1?" Human Mutation, vol. 9, no. 5, pp. 458-464, 1997.

[11] A. S. Rohl, E. Kahen, S. J. Yoder, J. K. Teer, and D. R. Reed, "The genomic landscape of malignant peripheral nerve sheath tumors: diverse drivers of Ras pathway activation," Scientific Reports, vol. 7, Article ID 14992, 2017.

[12] E. Martin, I. Asem, D. J. Grunhagen, J. V. M. G. Bovee, and C. Verhoef, "Prognostic significance of immunohistochemical biomarkers and genetic alterations in malignant peripheral nerve sheath tumors: a systematic review," Frontiers in Oncology, vol. 10, Article ID 594069, 2020.

[13] T. De Raedt, H. Brems, P. Wolkenstein et al., "Elevated risk for MPNST in NF1 microdeletion patients," The American Journal of Human Genetics, vol. 72, no. 5, pp. 1288-1292, 2003.

[14] G. Spurlock, S. Griffiths, J. Uff, and M. Upadhyaya, "Somatic alterations of the NF1 gene in an NF1 individual with multiple benign tumours (internal and external) and malignant tumour types," Familial Cancer, vol. 6, no. 4, pp. 463-471, 2007.

[15] S. L. Carroll, "The challenge of cancer genomics in rare nervous system neoplasms," American Journal Of Pathology, vol. 186, no. 3, pp. 464-477, 2016.

[16] C. R. Abernathy, S. A. Rasmussen, H. J. Stalker et al., "NF1 mutation analysis using a combined heteroduplex/SSCP approach," Human Mutation, vol. 9, no. 6, pp. 548-554, 1997.
[17] H. Li, L.-J. Chang, D. R. Neubauer, D. F. Muir, and M. R. Wallace, "Immortalization of human normal and NF1 neurofibroma Schwann cells," Laboratory Investigation, vol. 96, no. 10, pp. 1105-1115, 2016.

[18] C. Zou, K. D. Smith, J. Liu et al., "Clinical, pathological, and molecular variables predictive of malignant peripheral nerve sheath tumor outcome," Annals of Surgery, vol. 249, no. 6, pp. 1014-1022, 2009.

[19] D. E. Porter, V. Prasad, and L. Foster, "Survival in malignant peripheral nerve sheath tumours: a comparison between sporadic and neurofibromatosis type 1-associated tumours," Sarcoma, vol. 2009, Article ID 756395, 5 pages, 2009.

[20] M. Anghileri, R. Miceli, M. Fiore et al., "Malignant peripheral nerve sheath tumors," Cancer, vol. 107, no. 5, pp. 1065-1074, 2006.

[21] S. Ingham, S. M. Huson, A. Moran, J. Wylie, M. Leahy, and D. G. R. Evans, "Malignant peripheral nerve sheath tumours in NF1: improved survival in women and in recent years," European Journal of Cancer, vol. 47, no. 18, pp. 2723-2728, 2011.

[22] C.-C. H. Stucky, K. N. Johnson, R. J. Gray et al., "Malignant peripheral nerve sheath tumors (MPNST): the Mayo Clinic experience," Annals of Surgical Oncology, vol. 19, no. 3, pp. 878-885, 2012.

[23] O. Goertz, S. Langer, D. Uthoff et al., "Diagnosis, treatment and survival of 65 patients with malignant peripheral nerve sheath tumors," Anticancer Research, vol. 34, no. 2, pp. 777-783, 2014.

[24] J. Kahn, A. Gillespie, M. Tsokos et al., "Radiation therapy in management of sporadic and neurofibromatosis type 1-associated malignant peripheral nerve sheath tumors," Frontiers in Oncology, vol. 4, p. 324, Article ID 324, 2014.

[25] T. Valentin, A. Le Cesne, I. Ray-Coquard et al., "Management and prognosis of malignant peripheral nerve sheath tumors: the experience of the French Sarcoma Group (GSF-GETO)," European Journal of Cancer, vol. 56, pp. 77-84, 2016.

[26] S. Le Guellec, A.-V. Decouvelaere, T. Filleron et al., "Malignant peripheral nerve sheath tumor is a challenging diagnosis," The American Journal of Surgical Pathology, vol. 40, no. 7, pp. 896-908, 2016

[27] K. L. Watson, G. A. Al Sannaa, C. M. Kivlin et al., "Patterns of recurrence and survival in sporadic, neurofibromatosis type 1associated, and radiation-associated malignant peripheral nerve sheath tumors," Journal of Neurosurgery, vol. 126, no. 1, pp. 319-329, 2017.

[28] I. K. Hwang, S. M. Hahn, H. S. Kim et al., "Outcomes of treatment for malignant peripheral nerve sheath tumors: different clinical features associated with neurofibromatosis type 1," Cancer Research and Treatment, vol. 49, no. 3, pp. 717-726, 2017.

[29] M. Carli, A. Ferrari, A. Mattke et al., "Pediatric malignant peripheral nerve sheath tumor: the Italian and German soft tissue sarcoma cooperative group," Journal of Clinical Oncology, vol. 23, no. 33, pp. 8422-8430, 2005.

[30] E. Martin, J. H. Coert, U. E. Flucke et al., "A nationwide cohort study on treatment and survival in patients with malignant peripheral nerve sheath tumours," European Journal of Cancer, vol. 124, pp. 77-87, 2020.

[31] J. S. Nix, M. C. Haffner, S. Ahsan et al., "Malignant peripheral nerve sheath tumors show decreased global DNA methylation," Journal of Neuropathology \& Experimental Neurology, vol. 77, no. 10, pp. 958-963, 2018.

[32] K. Yang, W. Guo, T. Ren et al., "Knockdown of HMGA2 regulates the level of autophagy via interactions between MSI2 
and Beclin1 to inhibit NF1-associated malignant peripheral nerve sheath tumour growth," Journal of Experimental \& Clinical Cancer Research: Climate Research, vol. 38, no. 1, p. 185, 2019.

[33] H. Kehrer-Sawatzki, V.-F. Mautner, and D. N. Cooper, "Emerging genotype-phenotype relationships in patients with large NF1 deletions," Human Genetics, vol. 136, no. 4, pp. 349-376, 2017.

[34] L. Kluwe, R. E. Friedrich, M. Peiper, J. Friedman, and V. F. Mautner, "Constitutional NF1 mutations in neurofibromatosis 1 patients with malignant peripheral nerve sheath tumors," Human Mutation, vol. 22, no. 5, p. 420, 2003.

[35] I. Bottillo, T. Ahlquist, H. Brekke et al., "Germline and somaticNF1mutations in sporadic and NF1-associated malignant peripheral nerve sheath tumours," The Journal of Pathology, vol. 217, no. 5, pp. 693-701, 2009. 